\title{
Classification of Steps on Road Surface Using Acceleration Signals
}

\author{
Extracting and Analyzing Road Information
}

\author{
Junji Takahashi \\ Aoyama Gakuin University \\ 1-10-5, Fuchinobe, Chuo-ku, \\ Kanagawa, Japan \\ takahashi@it.aoyama.ac.jp
}

\author{
Yusuke Kobana \\ Graduate School of Aoyama \\ Gakuin University \\ 1-10-5, Fuchinobe, Chuo-ku, \\ Kanagawa, Japan \\ ykobana@wil-aoyama.jp \\ Guillaume Lopez \\ Aoyama Gakuin University \\ 1-10-5, Fuchinobe, Chuo-ku, \\ Kanagawa, Japan \\ guillaume@wil- \\ aoyama.jp
}

\author{
Yoshito Tobe \\ Aoyama Gakuin University \\ 1-10-5, Fuchinobe, Chuo-ku, \\ Kanagawa, Japan \\ yoshito-tobe@rcl- \\ aoyama.jp
}

\begin{abstract}
In order to reduce a road monitoring cost, we propose a system to monitor extensively road condition by cyclists with a smartphone. In this paper, we propose two methods towards road monitoring. First is to classify road signals to four road conditions. Second is to extract road signal from a smartphone's accelerometer in three positions: pants' side pocket, chest pocket and a bag in a front basket. In pants' side pocket, road signal is extracted by Independent Component Analysis. In chest pocket and bag in a front basket, road signal is extracted by selecting 1 -axis affected from gravitational acceleration. In the experiment of the classification method, overall accuracy was $75 \%$. The experimental results of the extraction methods with correlation coefficient showed the overall accuracy were more than 0.7 in pants' side pocket and chest pocket, the overall accuracy was less than 0.3 in bag in a front basket.
\end{abstract}

\section{Categories and Subject Descriptors}

D.2 [Computer Applications]: Physical sciences and engineering; I.5.4 [Computing Methodologies]: Applications-Signal processing

\section{General Terms}

Algorithms, Measurement, Experimentation, Verification

\section{Keywords}

Wearable sensor, Road monitoring, Independent Component Analysis

\section{INTRODUCTION}

There has been much attention to the maintenance of road infrastructure in Japan since Sasago Tunnel accident in 2012. Detection of possible failure and already damaged points is important, but it needs special-purpose measurement equipment. Moreover, road infrastructures that should be monitored are enormous. Therefore, an easier way of detecting damaged points is anticipated.

To satisfy the goal, crowdsensing can be a candidate for road sensing; it is realized by combining sensing technologies and the Web. Sensing workers collaborate on the Web for a sensing task. Based on the idea of crowdsensing, we focus on road sensing utilizing mobile phones carried by people riding bikes. Using bikes is becoming promising because bike sharing is popular in many cities and personal bikes are also increasing.

There has been several works on utilization of bikes such as BikeNet [1] and Probe Bicycle. These works assume that bikes are equipped with necessary sensors and we need uniform special-purpose bikes everywhere. In contrast, our system called YKOB (Your Kinetic Observation Bike) needs only users' smartphones. In our earlier work, we limited ourselves to a case where a user puts a smartphone in a pants' side pocket. However, we need to allow users to put their smartphone in other parts of their body to collect many participants.

In this paper, we have investigated two other cases of placement of a smartphone: a pocket of shirt and a bag in a basket attached to the bike. In this experiment, similarities of the collected acceleration signal between each places were evaluated by a correlation coefficient. The results of our experiment show that a pocket of shirt can contribute to classification in the same quality as a hip.

The rest of the paper is organized as follows: Section 2 describes related works. Sections 3 and 4 explain the experimental environment and the classification algorithm for a hip, respectively. Section 5 describes the results with the other two placements and section 6 concludes this work. 


\section{RELATED WORKS}

A research of participatory sensing has developed along with the spread of mobile terminals. As one of the pioneering research, WINFO [2] which acquires information of conditions of one's umbrella via cellular telephony network and visualizes area of sudden rains was proposed. Like this example, the system, that acquires various information obtained at one's cell-phone, analyzes them on a server and provides information of high value, was named "Participatory sensing" and its various problems were presented by Burke [3]. Thereafter this system has also been called as "Crowdsensing" to mean using crowdsourcing, and been developed for real world application through the discussion of coverage problem [4] or collaboration among nodes [5].

As cases of applying the participatory sensing for city environment survey, Noisemap [6,7], which utilizes the microphone data and GPS location data of smartphone, were proposed. Christin etal. reported thermal maps, atmosphere pollution maps, and traffic situation maps for monitoring city environment [8]. A study utilizing a public transportation system rather than person is reported. Hasenfratz etal. investigated radio field strength in Zurich city using trams [9].

Studies for monitoring road condition based on participatory sensing are reported. Erikkson etal. investigated road condition total length of $9730 \mathrm{~km}$ within Boston city by 7 cabs equipped with 3 -axis acceleration sensor and detected road anomaly in need of restoration [10]. Mohan etal. collected acceleration data of smartphone that a person wears during bus ride or train ride, then estimated road condition by extracting vehicle acceleration based on the direction difference between vehicle and smartphone [11]. However, the former has a problem in monitoring of large area and long terms due to specialized devices, the latter has a problem in detection accuracy. Yagi proposed a road monitoring method using a smartphone and vehicle which overcomes both of large-long scale monitoring and detection accuracy. In his method, the smartphone is located on dashboard of the vehicle and records acceleration data to detect road anomaly. His system succeeded to detect a step of $2.4 \mathrm{~cm}[12]$.

Studies using bike for monitoring road condition are reported. A bike is light comparing with a car and usually does not have a suspension, so it can detect small step even if its speed is slow. Moreover, the bike can enter a narrow street that a car cannot so that the monitoring system can cover area closely. Eisenman etal. proposed BikeNet which monitors cyclist experience and makes a map with several sensors mounted on the bike [1]. This system needs to equip sensors on bie body so that there is a difficulty to increase participants. Reddy etal. proposed data sharing platform named Biketastic which enriches experimentation of finding good routes and sharing route information among cyclists. This system does not need specialized sensors equipped on the bike but only need smartphone to be worn by the cyclist [13]. This system evaluates the route in terms of safety, efficiency, and enjoyment. Verstockt etal. proposed a method to categorize the road type using data collected at worn smartphone of cyclist [14]. Their algorithm receives 5 seconds of acceleration data and calculates their magnitude and then classifies it into 6 road terrain types based on random forest of binary decision trees. Therefore, although their proposed method can be utilized for macroscopic road terrain classification, it cannot be suited for microscopic road damage monitoring like a road damage detection.

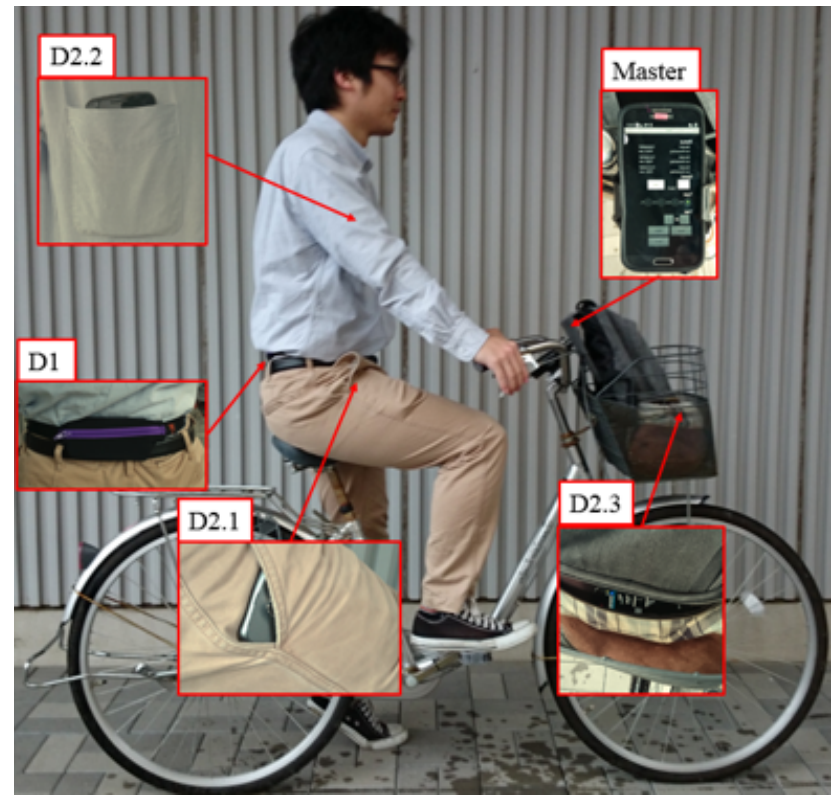

Figure 1: The experimental environment

\section{EXPERIMENTAL ENVIRONMENT}

We assume that cyclists carry their smartphone in mainly three positions: pants' side pocket, shirt pocket, and front basket bag of bike. In this paper, we experimentally approach to extracting road signal from these positions of smartphone. Figure 1 shows experimental environment. D1, D2.1, and D2.2 are a smartphone on the hip, in pants' side pocket, and in a shirt pocket, respectively. In order to collect acceleration signals from smartphones in these positions simultaneously, we use the smartphone placed in the handle (named Master in Figure 1) which is connected to all other smartphones with Bluetooth. The bike under the experiment is a city bike commonly used in Japan. The bike speed is controlled between 8 and $12 \mathrm{~km} / \mathrm{h}$ with SIGMA ROX5.0 which measures the speed by calculating the number of rotation of the front wheel. The sampling rate of accelerometer on the smartphones is set to $50 \mathrm{~Hz}$ for hardware reason.

\section{STEP CLASSIFYING ALGORITHM BASED ON ACCELERATION FROM THE HIP}

\subsection{Step classifying algorithm}

Classifying road signals is important to avoid misdetection of road damages. Therefore as a first step we classify four basic conditions: positive step damage (PS), negative step damage (NS), convex step damage (CS) and at flat status (FL) (See Figure 2). The step classifying algorithm is based on $\mathrm{x}$-axis acceleration affected by gravity. The signal is regarded as a road signal. Figure 3 shows an acceleration signal which moving average and centering are applied to. Table 1 shows the features of wheel state for each road condition. In the figure and table, a large peak appears when Collision or Landing of wheel from step. In case of negative step, negative acceleration change from Free-fall appears before peak of Collision. In addition, peaks appear twice because of front and rear wheel passing. On the other hand, a significant change of acceleration does not appear in the flat status. Considering these features, we propose our step classifying algorithm. 

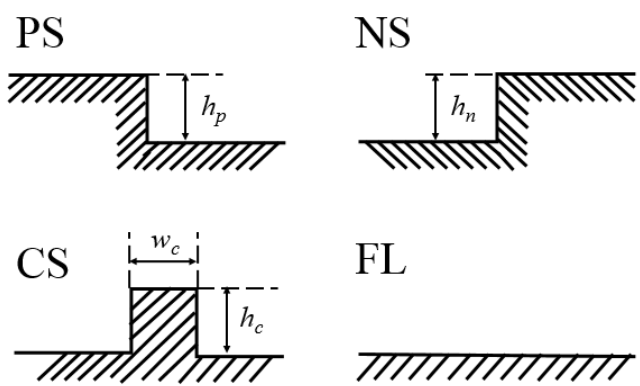

FL

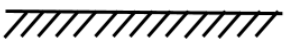

Figure 2: Four road conditions

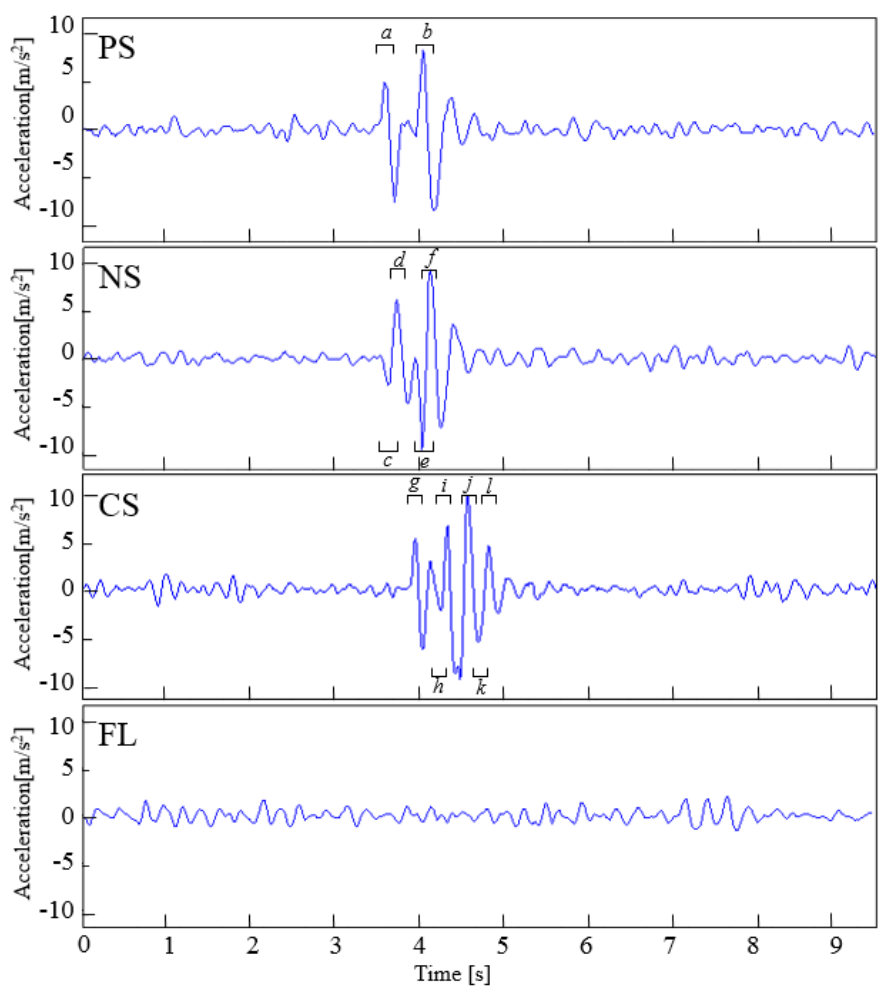

Figure 3: Acceleration signals in $x$-axis from smartphone on the hip

First the acceleration signal is classified whether flat or not using positive threshold $T_{\text {plus }}$. Then the signal of non-flat status is classified whether a negative step damage or not. Algorithm 1 shows an algorithm to classify a negative step damage. Let us define the time of exceeding $T_{\text {plus }}$ as $t_{\text {pstep }}$. In addition, let $t, t_{\text {pre }}$, and $A_{\text {minus }}$ be the elapsed time, the short time before $T_{p s t e p}$, and the amount of negative changing between $t_{\text {pstep }}$ and $t_{\text {pre }}$, respectively. This algorithm focuses on negative changing from Free-fall as seen at $\mathrm{c}$ and e in Figure 3. If $A_{m}$ inus exceeds negative threshold $M_{\text {thre }}$, the signal is classifed as negative step damage. Otherwise the signal is classified as non-negative step damage. Finally, the non-negative step damage is differentiated positive or convex step damage. Algorithm 2 shows an algorithm of classifying positive and convex step damages. The number of peaks $N_{\text {plus }}$ exceeding $T_{\text {plus }}$ is utilized to classify these steps. As can be seen in Table 1, in convex step damage $N_{\text {plus }}$ will be 4 . It is greater than positive step damage.
Table 1: Wheel state when passed each step

\begin{tabular}{cccccc}
\hline Wheel & State & PS & NS & CS & FL \\
\hline Front & Collision & $a$ & - & $g$ & - \\
& Free-fall & - & $c$ & $h$ & - \\
& Landing & - & $d$ & $i$ & - \\
\multirow{5}{*}{ Rear } & Collision & $b$ & - & $j$ & - \\
& Free-fall & - & $e$ & $k$ & - \\
& Landing & - & $f$ & $l$ & - \\
\hline
\end{tabular}

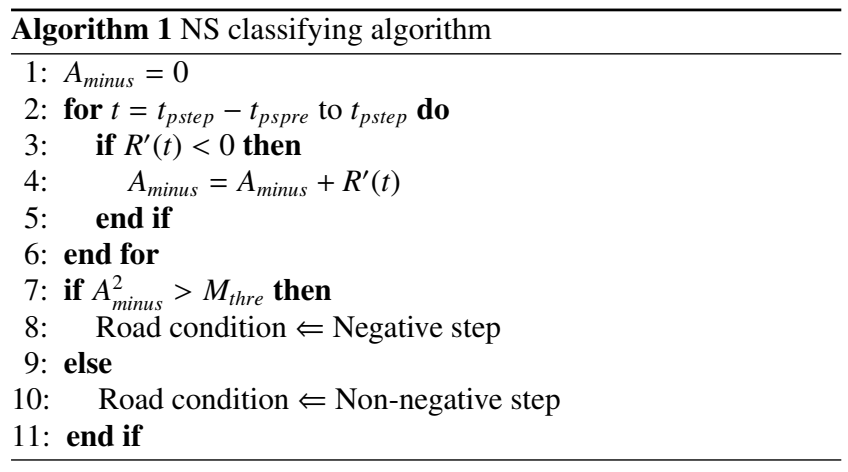

\subsection{Evaluating step classifying algorithm}

We evaluate step classifying algorithm. The number of passing each step was 20 times. The height of positive step $h_{p}$ and negative step $n_{p}$ were $46 \mathrm{~mm}$. The height of convex step $h_{c}$ was $53 \mathrm{~mm}$, width $h_{w}$ was $98 \mathrm{~mm}$. The parameter described in this algorithm is that $T_{\text {plus }}$ is $4.0, t_{\text {pre }}$ is $0.2, M_{\text {thre }}=-15$. Table 2 shows the result of this algorithm. The overall classification accuracy was $75 \%$. However the accuracy of classifying positive or convex step damage algorithm was relatively low. This could be caused by that acceleration signal could not be changed largely when Collision happened. Actually the amount of acceleration changing in Collision is larger than Landing. We will deal with this problem by setting smaller threshold after Collision than $T_{\text {plus }}$.

\section{MEASUREMENT AND ANALYSIS OF AC- CELERATION SIGNAL IN RESPECTIVE SMARTPHONE LOCATIONS}

We confirmed that the acceleration signal measured by the smartphone near the hip reflected road conditions properly in the above section. However, a cyclist rarely wear the smartphone on the hip but on the pants' side pocket, in the chest pocket or in the bag at the front of the bike. We experimentally measure the acceleration signals of smartphone in respective locations and analyze them by comparing with the signal measured on the hip. The number of measurement trials are 20 times per a road step. The data are collected synchronously using four smartphones in respective locations. They are connected to a master device fixed on the bike handle via Bluetooth to be operated synchronously by our original android application.

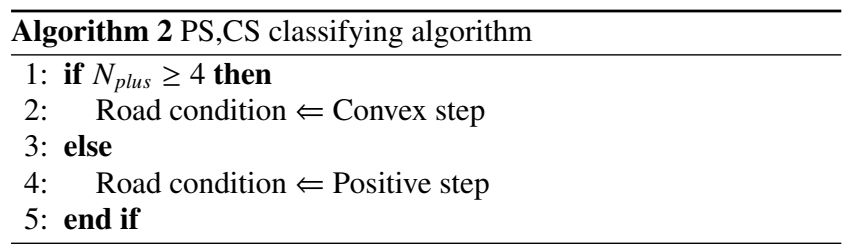


Table 2: The results of the classifying experiment

\begin{tabular}{l|c|rrrr|r}
\hline \multicolumn{2}{c|}{} & \multicolumn{5}{|c}{ Correct answer } \\
\cline { 3 - 7 } \multicolumn{2}{c}{} & PS & NS & CS & FL & Recall \\
\hline \multirow{4}{*}{ Estimated } & PS & 15 & 1 & 6 & 0 & 0.68 \\
& NS & 1 & 16 & 0 & 0 & 0.94 \\
& CS & 4 & 3 & 14 & 0 & 0.67 \\
& FL & 0 & 0 & 0 & 20 & 1.00 \\
\hline \multicolumn{2}{c|}{ Accuracy } & 0.75 & 0.80 & 0.70 & 1.00 & \\
\hline
\end{tabular}

Figure 4 shows examples of collected data including acceleration signal in $\mathrm{x}, \mathrm{y}, \mathrm{z}$-axis. The significant variations around 8 seconds imply that the bike overpassed a step at that time. First, we evaluate how well the acceleration signal represents the road condition. For this purpose, we assume the acceleration signal of $\mathrm{x}$-axis at the hip, which directs the gravity-direction, is true signal, and calculate the coefficient of correlation between the true signal and a target signal. In fact, the acceleration data collected on respective locations has time differences in a precise sense in spite of synchronized operation. In order to eliminate the time differences for proper calculation, the time offset $\tau(-1.0<\tau<1.0)$ is used, and the formulation is given as follows,

$$
\begin{aligned}
C(\tau) & =\frac{\sum_{t=0}^{T}\left(r_{(t)}-\bar{r}\right)\left(y_{(t+\tau)}-\bar{y}\right)}{\sqrt{\sum_{t=0}^{T}\left(r_{(t)}-\bar{r}\right)^{2}} \sqrt{\sum_{t=0}^{T}\left(y_{(t+\tau)}-\bar{y}\right)^{2}}}, \\
C & =\max C(\tau) .
\end{aligned}
$$

Where, the $r(t)$ denotes the true signal and $y(t)$ denotes the target acceleration signal. Then the correlation $C$ between two signals is given as maximum of the $C(\tau)$. If the $C$ is big enough, then it means that the target signal reflects road conditions properly and that the our proposed classifying step algorithm can be utilized for recognizing road condition. The details are described in following sections in respective smartphone locations.

\subsection{Pocket on chest}

The acceleration signals of $\mathrm{x}, \mathrm{y}, \mathrm{z}$-axis are shown at lower left in Figure 4. The calculation results of the $C(\tau)$ between the true signal and the $\mathrm{x}, \mathrm{y}, \mathrm{z}$-axis acceleration signals are shown in Figure 5. Clearly the $C(\tau)$ of y-axis acc. is biggest and the $\mathrm{C}$ is 0.79 in this case. The average $\mathrm{C}$ of each step are $C_{\overline{p s}}=0.77, C_{\overline{n s}}=0.85$, $C_{\overline{p s}}=0.82$. Generally it is said that when the coefficient of correlation is bigger than 0.7, there is a strong correlation. Briefly the $y$-axis acc. reflects the road condition properly and can be utilized for classifying road steps.

\subsection{Pants' side pocket}

The $\mathrm{x}, \mathrm{y}, \mathrm{z}$-axis acceleration signals are shown on upper right in Figure 4. The y-axis acc. signal is affected by cyclist's pedaling motion. So, a preprocessing that extracts road signal from mixed signal is needed before step classification.

We proposed to apply the Independent Component Analysis (ICA) for extracting road signal. The ICA is a method that obtains original signals by estimating mixing process from input multiple observed signals [15]. Let $t$ denote time, $\boldsymbol{x}=\left(x_{1}(t), x_{2}(t), \cdots, x_{n}(t)\right)$

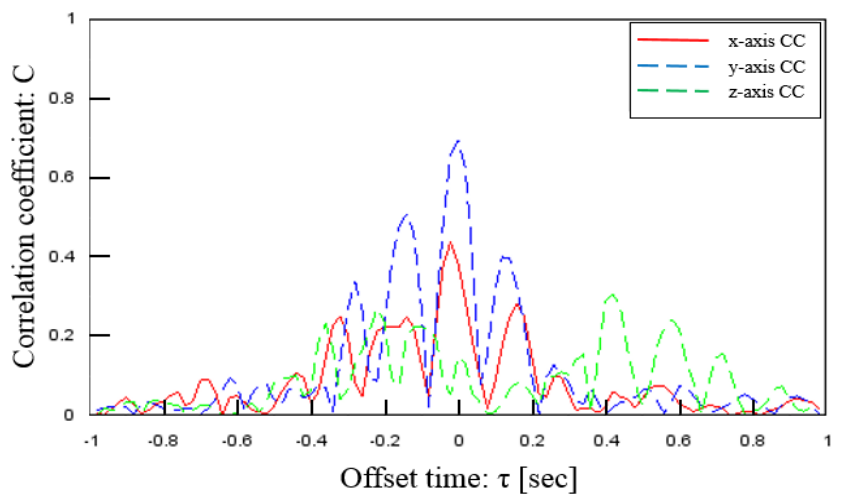

Figure 5: Correlation between true signal and signals from smartphone on the chest

denote observed signals, $\boldsymbol{W}$ denote disaggregation matrix, then the original independent signals $\boldsymbol{y}=\left(y_{1}(t), y_{2}(t), \cdots, y_{n}(t)\right)$ are given as follows,

$$
\boldsymbol{y}(t)=\boldsymbol{W} \boldsymbol{x}(t) .
$$

The disaggregation matrix $\boldsymbol{W}$ is determined to maximize the statistical independence by iterative calculation. In this paper, we use a kurtosis as a benchmark for the statistical independence and the FastICA algorithm [16] for iterative calculation. The FastICA algorithm usually outputs multiple independent signals. Based on our pilot-analysis, we decide that the signal which has highest kurtosis is the road signal and the one with second highest kurtosis is the pedaling signal. Fig.6 shows a result of signal by applying ICA to collected signals. The results of ICA calculation is shown in Figure 6 . The average $\mathrm{C}$ after ICA calculation are $C_{\overline{p s}}=0.71, C_{\overline{n s}}=0.73$, $C_{\overline{p s}}=0.74$. Therefore it is confirmed that the road signal is properly extracted from the signals collected in the pants' side pocket by ICA calculation.
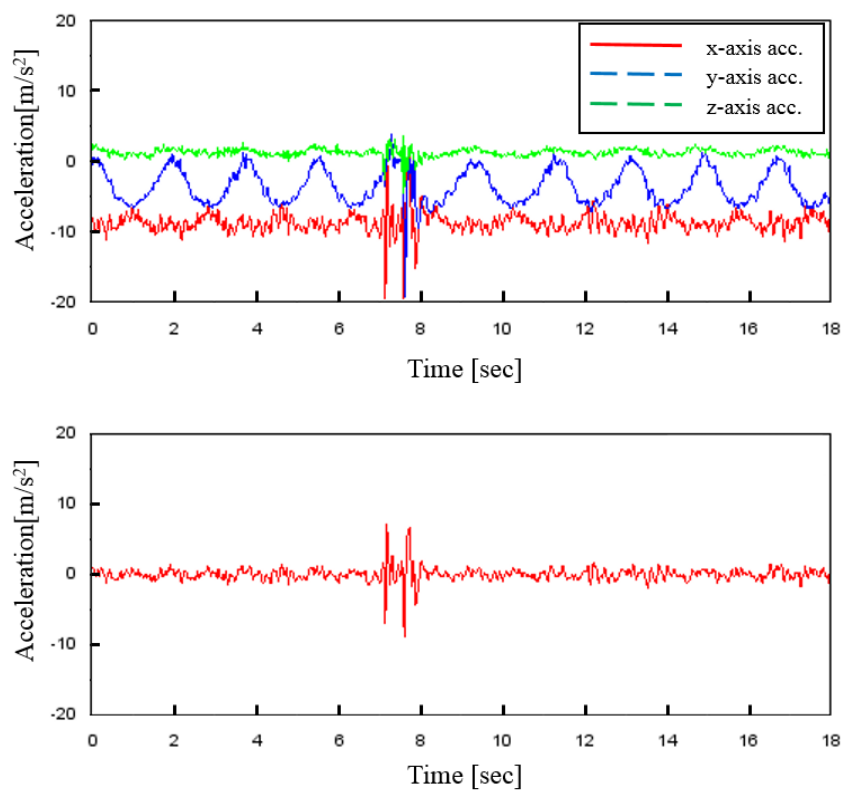

Figure 6: Observed signals from smartphone in the pockcet of trousers(upper) and extracted signal by applying ICA(lower) 

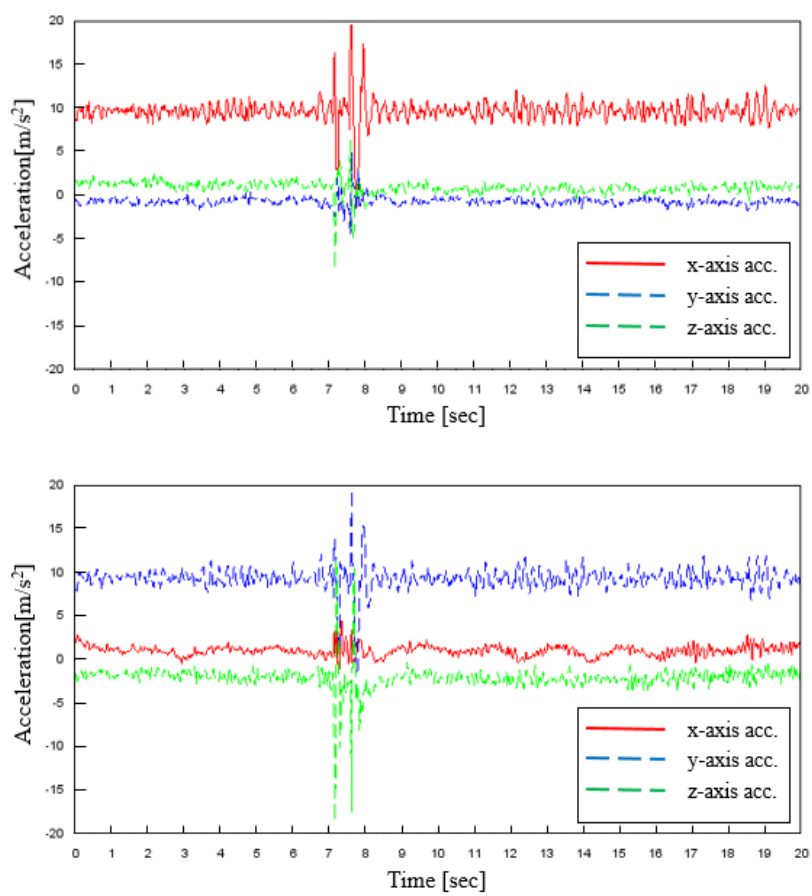
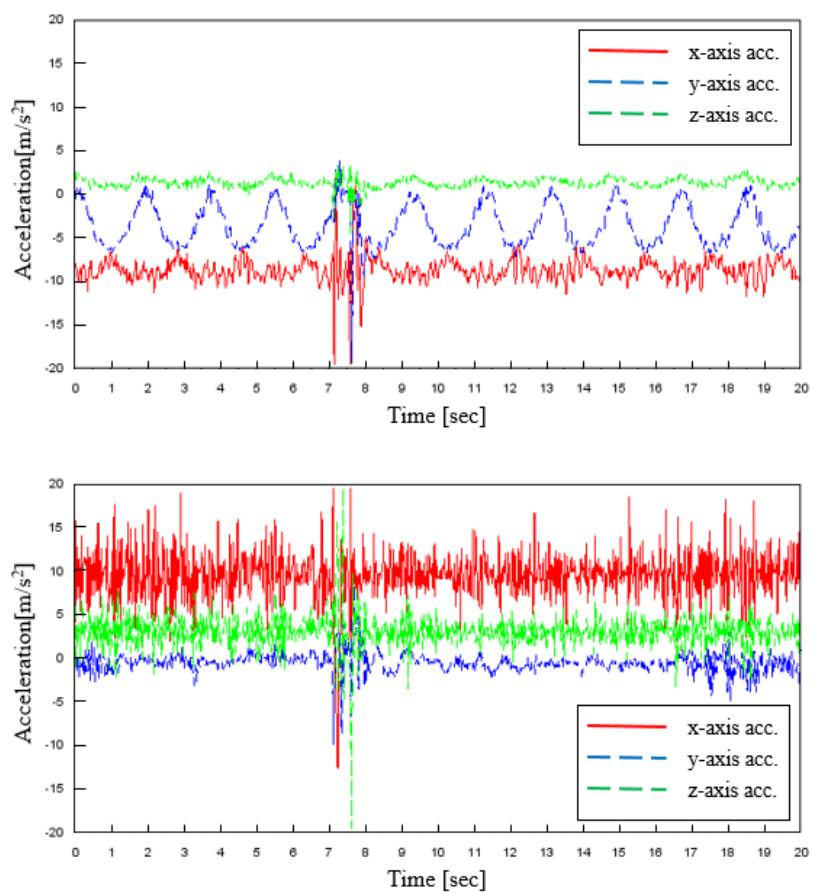

Figure 4: Acceleration signals from smartphone in four positions: hip(upper left), pants' side pocket(upper right), shirt pocket(lower left), bag in the front basket(lower right)

\subsection{Bag at the front of the bike}

The acceleration signals of $\mathrm{x}, \mathrm{y}, \mathrm{z}$-axis are shown at lower right in Figure 4. The calculation results of the $C(\tau)$ between the true signal and the $\mathrm{x}, \mathrm{y}, \mathrm{z}$-axis acceleration signals are shown in Figure 7. The $\mathrm{x}$-axis acceleration, which is in the direction of gravity, is thought to include largest information of road signal. Using this signal, the average $\mathrm{C}$ of each step are $C_{\overline{p s}}=0.29, C_{\overline{n s}}=0.15, C_{\overline{p s}}=0.30$.

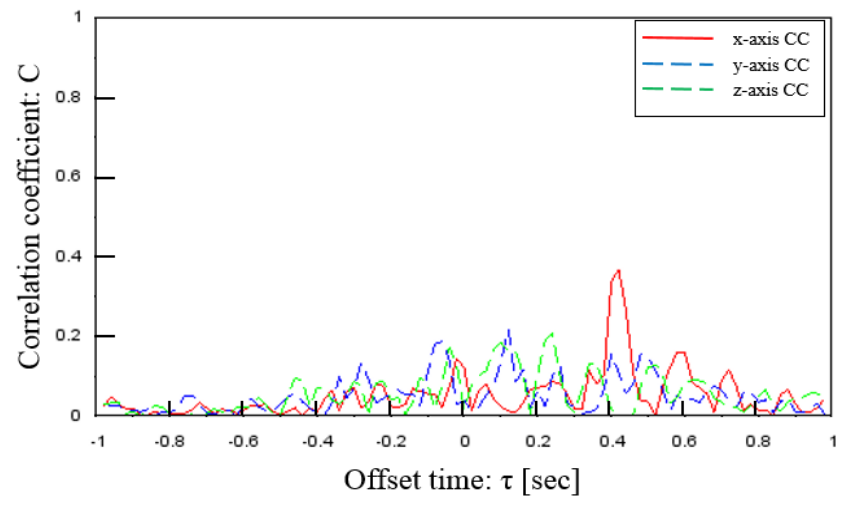

Figure 7: Correlation between true signal and signals from smartphone on the bag

The graph shows the signals are not affected by cyclist's motion. However, the coefficient of correlation is low. This is because the smartphone in the front basket of the bike is strongly affected by front wheels of the bike, contrastively the smartphone in the chest pocket and in the pants' side pocket is strongly affected by rear wheel. Therefore another step classifying algorithm is needed when the acceleration. Collected in the front basket is utilized for estimating road condition.

\section{CONCLUSION}

This paper has presented an algorithm to classify steps in a road surface using $\mathrm{x}$-axis acceleration of a smartphone in a waste pocket. The accuracy of classification turns out to be $75 \%$. It has also proposed other methods of classification using a pocket of shirt and a basket of bike. We have compared the three locations in terms of accuracy of classification and the results show that the both pockets indicates good correlation at 0.7 with the different steps and they are expected to be applied to the step classification algorithm using $\mathrm{x}$-axis acceleration.

Our future works include enhancing robustness of the algorithm with more users. In particular, the users may put their smartphones in many locations in addition to the three categories in this paper. Further extensive study is being planned.

\section{REFERENCES}

[1] Eisenman, B. Shane, Miluzzo, E., Lane, N. D., Peterson, R. A., Ahn G.S., and Campbell, A. T.: BikeNet: A mobile sensing system for cyclist experience mapping. ACM. Trans. Sensor Networks, Vol.1, No.6, (2009.12).

[2] Sasaki, K., Inoue, U., Tobe, Y.,“ WINFO: A human-assisted sensor network," Proc. of International Workshop on Networked Sensing Systems, 2005

[3] Burke, J., Estrin, D., Hansen, M., Parker, A., Ramanathan, N., Reddy, S., and Srivastava, M. B., "Participatory sensing," Proc. of Workshop on World-Sensor-Web (WSW'06): Mobile Device Centric Sensor Networks and Applications, (2006).

[4] Ahmed, A., Yasumoto, K., Yamauchi, Y., Ito, M. : Probabilistic Coverage Methods in People-Centric Sensing. JIP 19: pp. 473-490 (2011).

[5] Thepvilojanapong, N., Konomi, S., and Tobe, Y.: A study of 
cooperative human probes in urban sensing environments. IEICE Trans. on Comm., vol. E93-B, No.11, pp.2868-2878(2010.10).

[6] Stevens, M., D'Hondt, E.: Crowdsourcing of Pollution Data using Smartphones, UbiComp'10 (2010.9).

[7] Meurisch, C. and Planz, K.: Noisemap - discussing scalability in participatory sensing, ACM SenseMine' 13 (2013.11).

[8] Christin, D., Reinhardt, A., Kanhere, S., and Hollick, M.: A survey on privacy in mobile participatory sensing applications, Journal of systems and software (2011).

[9] Hasenfratz, D., Sturzenegger, S., Saukh, O., and Thiele, L.: Spatially resolved monitoring of radio-frequency electromagnetic fields, ACM SenseMine' 13 (2013.11).

[10] Eriksson, J., Girod, L., Hull B., Newton, R., Madden S., Balakrishnan, H., "The Pothole Patrol: Using a Mobile Sensor Network for Road Surface Monitoring," Proc. of The Int. Conf. on Mobile Systems, Applications, and Services, 2008.

[11] Prashanth, M., Padmanabhan, V. N., and Ramjee, R. Nericell: rich monitoring of road and traffic conditions using mobile smartphones, ACM SenSys'08, pp. 323-336 (2008).

[12] K. Yagi, "Extensional Smartphone Probe for Road Bump Detection," Proc. of the 17th ITS World Congress, 2010

[13] S. Reddy, K. Shilton, G. Denisov, C. Cenizal, D. Estrin, M Srivastava, "Biketastic: Sensing and Mapping for Better Biking," Proc. of SIGCHI Conference on Human Factors in Computing Systems, 2010 This paper proposes a platform that enriches experimentation and route sharing process.

[14] S. Verstockt, V. Slavkovikj, P.D. Potter, and R.V. Walle, "Collaborative Bike Sensing for Automatic Geographic Enrichment," IEEE SIGNAL PROCESSING MAGAZINE, 2014

[15] Hyvärinen, A. Karhunen, J. and Oji, E.: Independent Component Analysis, John Wiley \& Sons(2001).

[16] Hyvärinen, A., Oja, Erkki,. "A Fast Fixed-Point Algorithm for Independent Component Analysis," Neural Computation, vol.9, no.7, pp.1483-1492, 1997. 